CORRIGENDUM

\title{
Novel vaccine vectors for HIV-1
}

Dan H. Barouch \& Louis J. Picker

Nature Reviews Microbiology 12, 765-771 (2014)

On page 768 of the article, the text has been changed to clarify that all vaccinated and control animals were challenged until documentation of SIV infection by virological and/or immunological criteria.

Furthermore, on page 768 , the text has been changed to specify both the number of vaccinated controllers shown in

Figure 2 after intrarectal challenge as well as the number of controllers after both intrarectal and intravaginal challenge.

Finally, the legend of Figure 2 has been changed to clarify that the graph shows intrarectal SIVmac239 challenge of

monkeys that have either been vaccinated twice with a strain 68-1 RhCMV/SIV vector (week 0 and week $14 ; n=36$ ) or once with these vectors (week 0 ) and were then vaccinated at week 14 with Ad5 vectors $(n=12)$. Changes have been made to both

the HTML and PDF versions of the article. The authors apologize to readers for any confusion caused. 